\title{
Atypical Signs of Compartment Syndrome Caused by Pressure Injury in Forearm
}

\author{
Tae Hyung Kim $^{1}$ (D), Hwan Jun Choi $^{2}$ (D), Syeo Young Wee ${ }^{1}$ (D) Jeong Jin Chun ${ }^{1}$ (D) \\ ${ }^{1}$ Department of Plastic and Reconstructive Surgery, Soonchunhyang University Gumi Hospital, Soonchunhyang University College of Medicine, Gumi; \\ ${ }^{2}$ Department of Plastic and Reconstructive Surgery, Soonchunhyang University Cheonan Hospital, Soonchunhyang University College of Medicine, Cheonan, Korea
}

\begin{abstract}
Acute compartment syndrome (ACS) is a serious complication which is usually associated with fractures. Herein, we report a case of a patient with volar forearm compartment syndrome associated with closed muscle rupture without fracture of which the only external signs were superficial contact burns and pain. A 21-year-old man presented to our emergency department with painful swelling in the right forearm after being caught in a machine. Although he presented with pain, no open wound or fracture was seen. The patient was presumed to have a contusion and discharged. He presented with worsening pain the next day, when typical clinical features of ACS were observed and emergency fasciotomy was performed. Intraoperatively, ruptured flexor muscles were noted. Delayed presentation of forearm compartment syndrome accompanied by closed muscle rupture without fracture is extremely rare. Diagnosis is not easy because severe pain could be the only clue and can mask the signs of compartment syndrome, at risk of being missed.
\end{abstract}

Keywords: Forearm; Compartment syndromes; Muscle diastasis; Surgical decompression

\section{Introduction}

Acute compartment syndrome (ACS) is a condition in which increased pressure within a closed fascial compartment limits circulation to tissues, sometimes resulting in tissue necrosis [1]. Swift diagnosis and treatment are necessary. In cases of traumatic injury, especially involving fractures, ACS is a possible diagnosis when typical symptoms appear [2]. However, it is often overlooked because the symptoms are frequently masked by pain.

As traumatic ACS is observed more often in lower extremities than in upper extremities [2], ACS of the forearm is uncommon, but it is still well recognized owing to several reports in the literature [3]. The etiology of ACS is known to be diverse. ACS is often accompanied by fractures in the affected limb [4]. Typically, symptoms exacerbate within a few hours after injury, making physicians strongly suspect ACS $[1,2]$. Herein, we report a rare case of delayed volar forearm ACS presented by a contact burn wound. It was associated with closed muscle rupture without fracture, a condition easily overlooked. A delay in presentation of typical symptoms resulted in an initially missed diagnosis, which could have led to catastrophic sequelae if ACS of the forearm had not been recognized the following day.

All procedures in our case report were performed in accordance with the ethical standards of the institutional research committee and with the 1964 Helsinki Declaration and its later amendments. The patient provided written informed consent for the publication and the use of his images.

Case Report

Received: September 9, 2020

Revised: November 21, 2020

Accepted: November 21, 2020

\section{Corresponding author:}

Hwan Jun Choi, M.D., Ph.D.

Department of Plastic and Reconstructive Surgery, Soonchunhyang University Cheonan Hospital,

Soonchunhyang University College of Medicine,

31 Suncheonhyang 6-gil, Dongnam-gu, Cheonan

31151, Korea

Tel: +82-41-570-2195

Fax: +82-41-574-6133

E-mail: iprskorea@gmail.com

This is an Open Access article distributed under the terms of the Creative Commons Attribution Non-Commercial License (https://creativecommons.org/licenses/by-nc/4.0/) which permits unrestricted non-commercial use, distribution, and reproduction in any medium, provided the original work is properly cited.

(c) 2021 Korean Wound Management Society 


\section{Case}

A 21-year-old man presented to our hospital's emergency department with painful swelling of his right forearm 3 hours after an injury. The patient's forearm had been caught in a conveyor belt and compressed for several minutes. Initial physical examination showed moderate swelling and tenderness in the forearm. He presented with some inability to move digits. No open wound was observed except superficial second-degree friction burns. No fracture was noted in the radiological evaluation. After conservative management, the pain had improved. The circulation of the patient's fingertips and forearm was normal; neither did he show any neurological symptoms. The patient was thus discharged with a presumptive diagnosis of contusion. He did not have any personal medical history, and his coagulation profile was within normal range.

However, he presented to our department the next day with worsening pain. The swelling of forearm had increased to a point where it felt tense. He also had a cold and tingling sensation in the forearm and hand. He was observed to have motor impairment, and contractures of all the digits with the wrist held in a flexed position (Fig. 1). Nevertheless, the patient's circulation was still fine, and all the fingertips and forearms were pinkish in color. Forearm ACS was strongly suspected and emergency fasciotomy was performed under general anesthesia. The time of diagnosis and treatment was close to 24 hours after the injury.
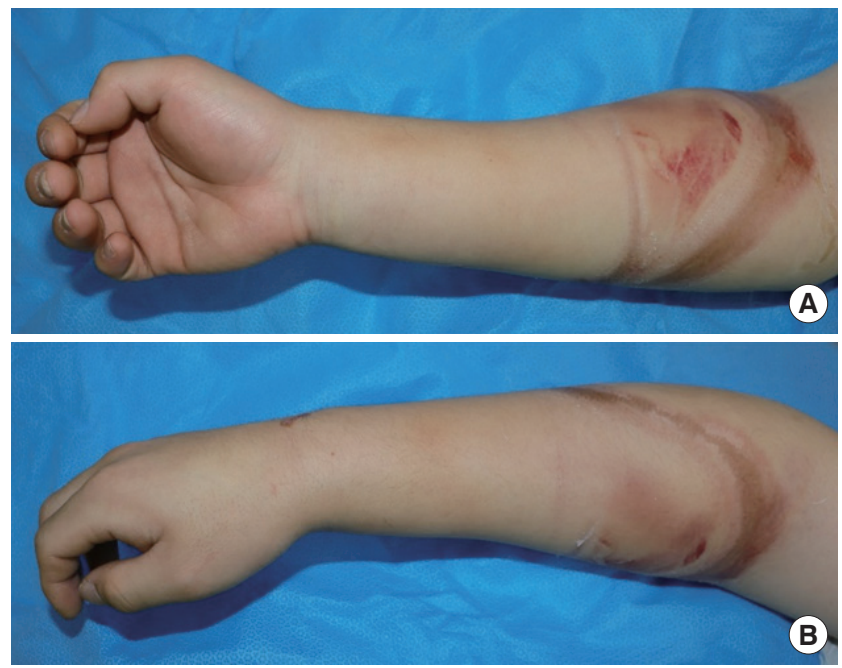

Fig. 1. Initial photographic findings of the patient. (A) The day after the injury, the patient presented with worsening pain and swelling. (B) Motor impairment and contractures of all digits with the wrist in a flexed position were observed.
A volar compartment fasciotomy was performed with a lazy-S patterned incision, $20 \mathrm{~cm}$ in length (Fig. 2). Intraoperative findings included approximately $50 \mathrm{~mL}$ of blood accumulated in a hematoma, and total rupture of the brachioradialis, palmaris longus, flexor carpi radialis and flexor digitorum superficialis muscles (Fig. 3A-C). Necrotic tissue was debrided. The color of the muscle belly was purplish due to ischemia (Fig. 3A and B). Evacuation of the hematoma and meticulous hemostasis were performed. A few minutes later, the color of the muscles had improved and changed to become reddish with better circulation (Fig. 3C). A myorrhaphy was executed after identifying the margins of the muscles and ensuring that they were not tattered. The incision was left open to avoid pressure. Retention sutures for prevention of skin edge retraction were applied (Fig. 3D). Negative pressure wound therapy was then performed to drain excess fluid from open wounds and reduce edema.

No necrotic change was observed in the muscle and the skin 10 days later and the initial friction burn had completely healed. Therefore, delayed primary closure was performed. The patient was discharged 3 weeks after the fasciotomy without recurrence of symptoms. His symptoms and motor function gradually improved. At the follow-up examination 4 months postoperatively, gross motor function was almost normal and contractures were not observed (Fig. 4). Electromyography and nerve conduction studies revealed incomplete injury of the median, radial, and ulnar nerves, with some evidence of regeneration. He did not suffer from any tingling sensation or cold.

\section{Discussion}

ACS is a surgical emergency because of the possibility of limbthreatening complications such as neurologic deficit, muscle

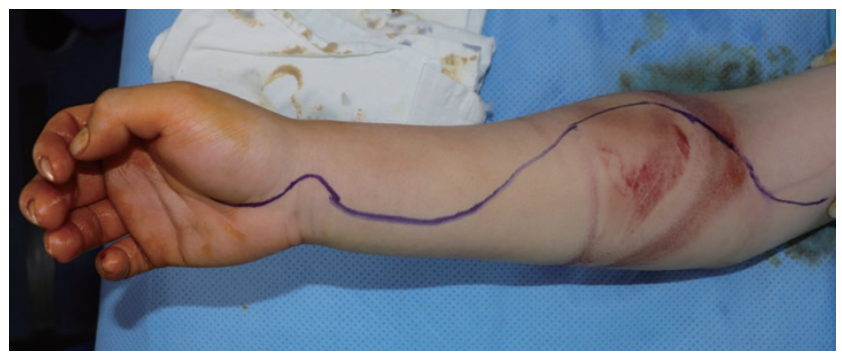

Fig. 2. The preoperative design of the forearm. A lazy-S patterned incision $20 \mathrm{~cm}$ in length was performed on the volar forearm for decompression. 

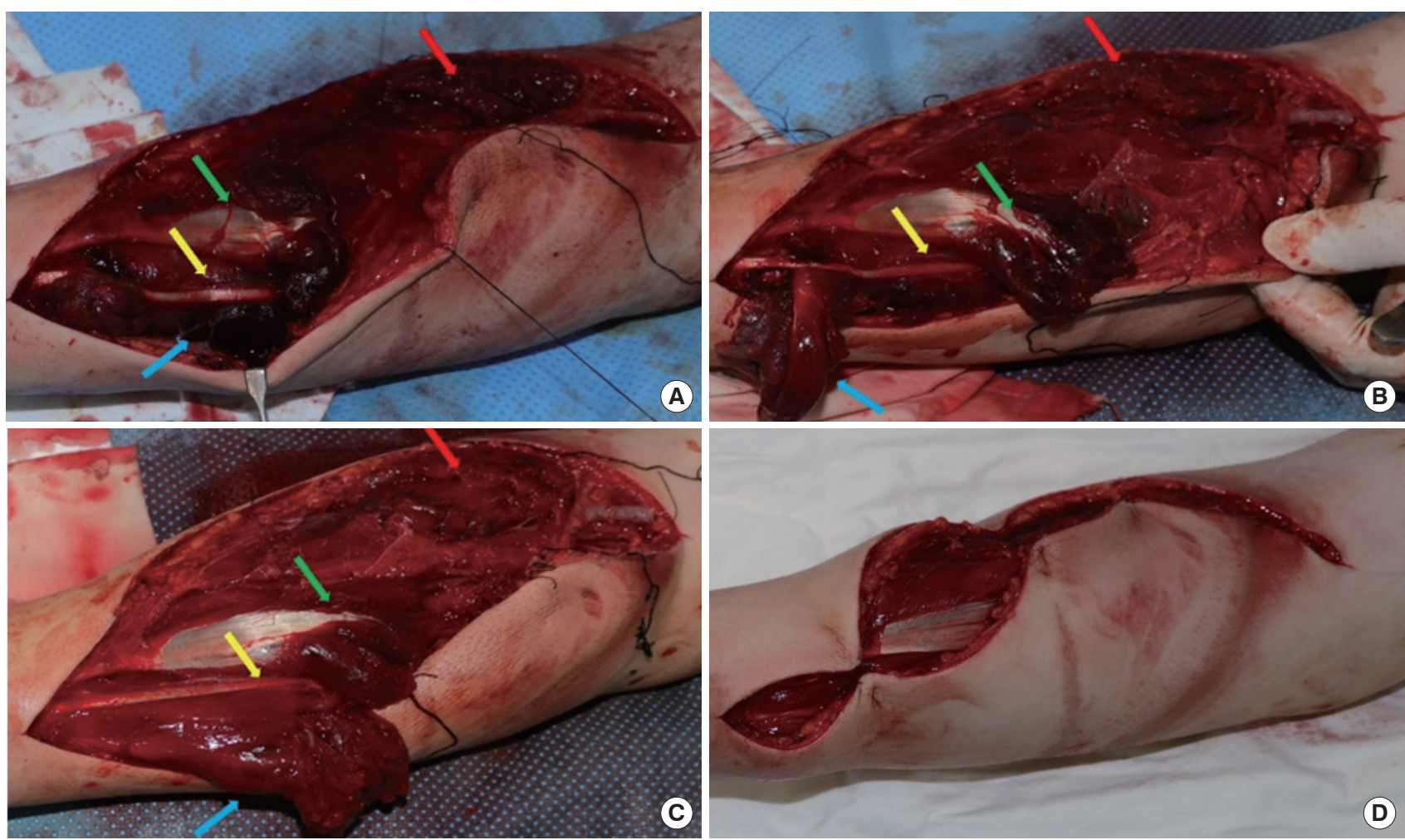

Fig. 3. Intraoperative photographic findings. (A) Complete rupture of the brachioradialis (red arrows), flexor carpi radialis (green arrows), palmaris longus (yellow arrows), and flexor digitorum superficialis (blue arrows) were discovered intraoperatively. (B) Change in the color of the muscles to dark purple due to decreased perfusion was observed. (C) A few minutes later after decompression, the color improved and became reddish. (D) The wound was left open. Retention sutures and negative pressure wound therapy were applied. Delayed wound closure was performed 10 days later.
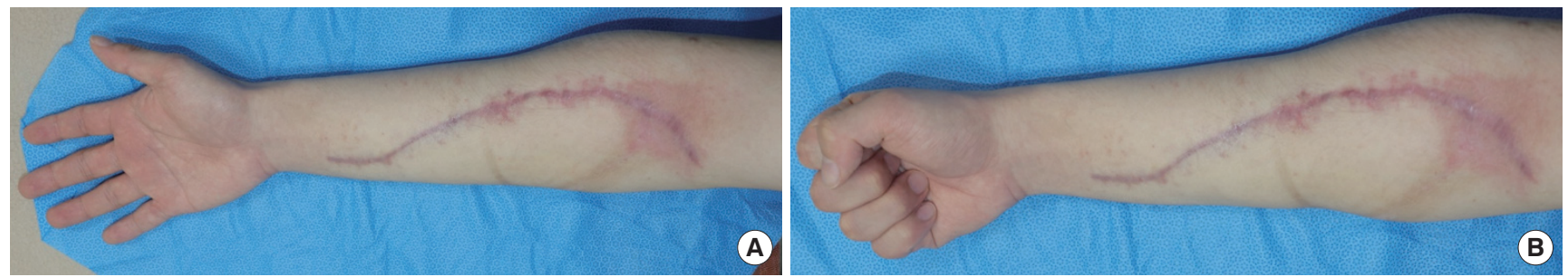

Fig. 4. Postoperative photographic findings. Movements of the digits and wrist including full extension (A) and full flexion (B) were restored. Gross motor function was almost normal and no contractures were observed at the 4 months postoperative follow-up.

necrosis, Volkmann ischemic contracture, infection, delayed healing of underlying fractures and rhabdomyolysis [1]. To avoid motor impairment secondary to these irreversible damages, aggressive surgical decompression via fasciotomy should be performed within six hours after an injury [5]. Therefore, prompt diagnosis and rapid decision-making are essential. The diagnosis of forearm ACS is usually based on clinical features. Traditionally, the hallmarks of diagnosis of ACS have been described as the six Ps: pain, pressure, paresthesia, pallor, paresis, and pulselessness [6]. The pain is usually out of pro- portion, and does not correlate with the external injury. This, along with the presence of pain on passive motion of the digits, are the most sensitive signs of ACS [3]. However, sensitivity of the clinical diagnosis is reported to be $13 \%-19 \%$ [7]. Furthermore, excessive pain makes it difficult to evaluate motor function during the acute phase of ACS [2].

In this case, the diagnosis was missed initially because the patient did not show typical symptoms of ACS except for severe pain and contact burn on the superficial dermal layer. There was also a delay in the onset of presentation of clinical 
features of forearm ACS. Even though intracompartmental pressure measurement provides objective data for diagnosis [3], it is not always required because ACS is a clinical diagnosis. In this case, further evaluation was omitted to decompress the pressure in the affected compartment as soon as possible, because more than 12 hours had passed since the injury. Although the diagnosis was initially missed, some residual doubt and a quick decision to perform a surgical decompression helped avoid catastrophic sequelae. Measuring intracompartmental pressure could have helped an accurate diagnosis in the first instance.

The etiology of forearm ACS is diverse; it may be induced by any type of injury. However, in approximately $70 \%$ of the patients it is accompanied by a fracture of the radius or ulna [4]. In contrast, ACS of the forearm caused by closed muscle rupture without fracture is rare. In a retrospective study, two among 16 patients with forearm ACS had closed muscle rupture without fracture [8]. Other studies exploring ACS of the forearm do not provide information about muscle disruption. The precise rate or etiology of muscle disruption induced-ACS has not been described. In this patient, it is believed that the muscles had ruptured due to high compression and traction.

To the extent of our knowledge, this is the first case report of forearm ACS in Korea caused by muscle rupture without an underlying fracture $[1,2,9]$. Though there is literature from overseas reporting similar case, this complication can still be overlooked due to the difficulty in distinguishing pain caused by ACS from that by contusion, the frequency of dermal contact burn by pressure injury, and rarity of the complication. Thus, we intend to highlight possible indicators of ACS. Surgeons should keep in mind that in cases of pressure injury without fracture, severe pain may suggest ACS with closed muscle rupture, even if the typical signs and symptoms are not present. Additionally, it is important to consider that even in the absence of a severe external injury, the internal structures may be seriously damaged. Overlooking severe pain and difficulty in movement of the digits may lead to irreversible complications. Clinicians should therefore keep alert for this rare type of ACS.

\section{Conflict of interest}

This work was supported by the National Research Founda- tion of Korea (NRF) grant funded by the Korea government (MSIT) (2020R1A2C1100891), and by Soonchunhyang University Research Fund. Otherwise, no potential conflict of interest relevant to this article was reported.

\section{ORCID iDs}

Tae Hyung Kim

https://orcid.org/0000-0001-9537-2330

Hwan Jun Choi

https://orcid.org/0000-0002-0752-0389

Syeo Young Wee

https://orcid.org/0000-0002-1787-9715

Jeong Jin Chun

https://orcid.org/0000-0001-6584-5782

\section{References}

1. Crawford B, Comstock S. Acute compartment syndrome of the dorsal forearm following noncontact injury. CJEM 2010;12:453-6.

2. Suzuki T, Takeda K, Iwamoto T, et al. Acute compartment syndrome in the forearm with extensor and flexor tendon ruptures: a case report. JBJS Case Connect 2014;4:e106.

3. Kistler JM, Ilyas AM, Thoder JJ. Forearm compartment syndrome: evaluation and management. Hand Clin 2018; 34:53-60.

4. Duckworth AD, McQueen MM. The diagnosis of acute compartment syndrome: a critical analysis review. JBJS Rev 2017;5:e1.

5. Elliott KG, Johnstone AJ. Diagnosing acute compartment syndrome. J Bone Joint Surg Br 2003;85:625-32.

6. Stevanovic MV, Sharpe F. Compartment syndrome and Volkmann ischemic contracture. In: Wolfe SW, Hotchkiss RN, Pederson WC, et al., editors. Green's operative hand surgery. 7th ed. Philadelphia: Elsevier; 2016. p. 1763-87.

7. Ulmer $\mathrm{T}$. The clinical diagnosis of compartment syndrome of the lower leg: are clinical findings predictive of the disorder? J Orthop Trauma 2002;16:572-7.

8. Suzuki T, Yamabe E, Iwamoto T, et al. Acute compartment syndrome of upper extremities with tendon ruptures. J Hand Surg Asian Pac Vol 2017;22:411-5.

9. Gainor BJ. Closed avulsion of the flexor digitorum superficialis origin causing compartment syndrome: a case report. J Bone Joint Surg Am 1984;66:467. 